\title{
Regulating Interfacial Coupling and Electron Transport for Efficient Electron Transporting Materials
}

Jiwei Tang, Kai Ren, Yan Zhang, Wei Shen, Rongxing He* and Ming Li*

Key Laboratory of Luminescence Analysis and Molecular Sensing (Southwest University), Ministry of Education, College of Chemistry and Chemical Engineering, Southwest University, Chongqing 400715, P. R. China

*Address correspondence to E-mail: herx@swu.edu.cn (Rongxing He), liming@swu.edu.cn (Ming Li). 


\section{Table captions}

Table S1 LUMO levels (eV) for NDI-ID at different functionals/6-31G $(\mathrm{d}, \mathrm{p})$ levels in dichloromethane

Table S1 LUMO levels (eV) for NDI-ID at different functionals/6-31G (d,p) levels in dichloromethane.

\begin{tabular}{cccccc}
\hline TPSS1KCIS & MPW1KCIS & B3LYP & MPW3LYP & PBE1PBE & Exp $^{\mathrm{a}}$ \\
\hline-4.00 & -4.07 & -4.30 & -4.32 & -4.57 & -4.10 \\
\hline
\end{tabular}

${ }^{a}$ Experimental value from Ref 1 .

\section{Figure captions}

Figure S1. The crystal structures for all studied molecules.

Figure S2. Independent gradient model (IGM) scatter plots of $\mathrm{MAPbI}_{3} / \mathrm{NDI}-\mathrm{ID}$, $\mathrm{MAPbI}_{3} / \mathrm{NFX}-\mathrm{ID}$ and $\mathrm{MAPbI}_{3} / \mathrm{NF} 2 \mathrm{X}-\mathrm{ID}$ interfaces.

Figure S3. The optimized geometries of the studied molecules at the B3LYP/6-31G (d, p) level.

Figure S4. The main stacking modes for all studied molecules.

Figure S5. Electrostatic potentials mapped on a surface of total electron densities. 


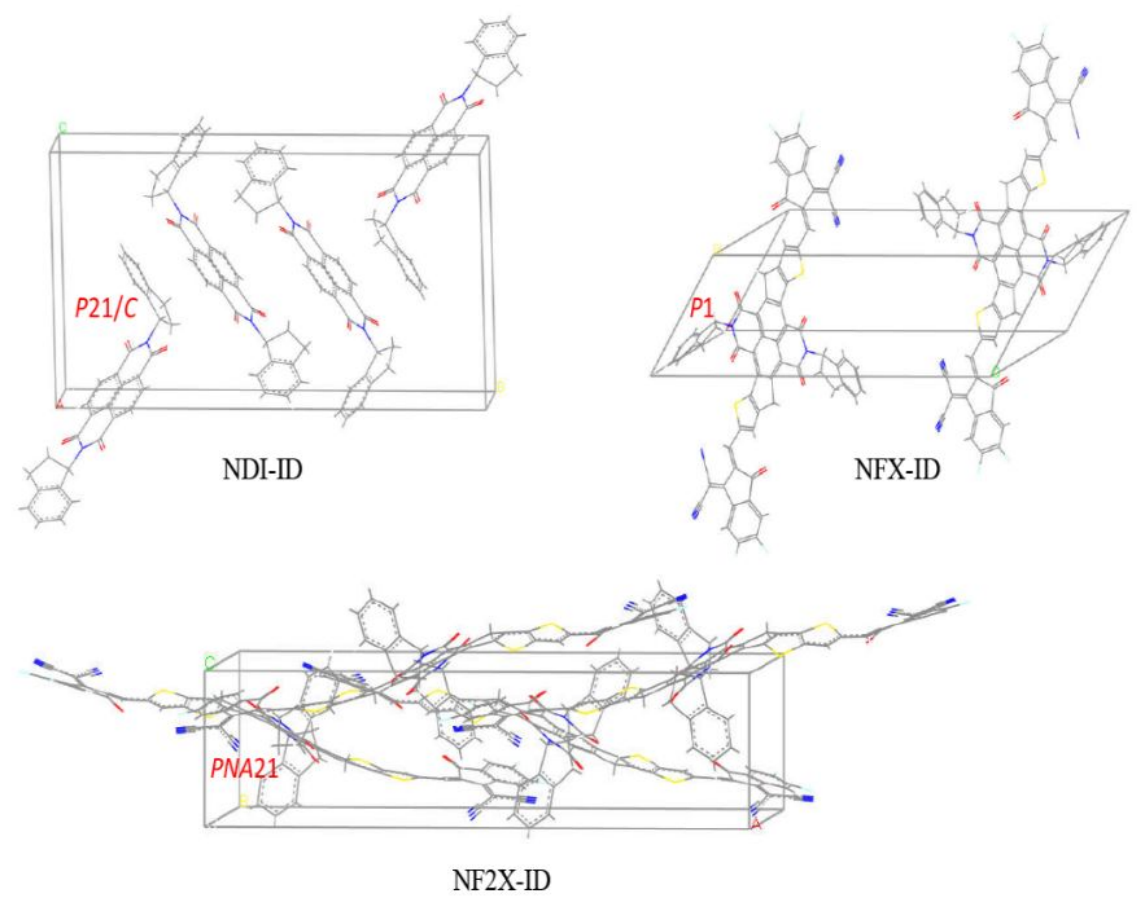

Figure S1 The crystal structures for all studied molecules.
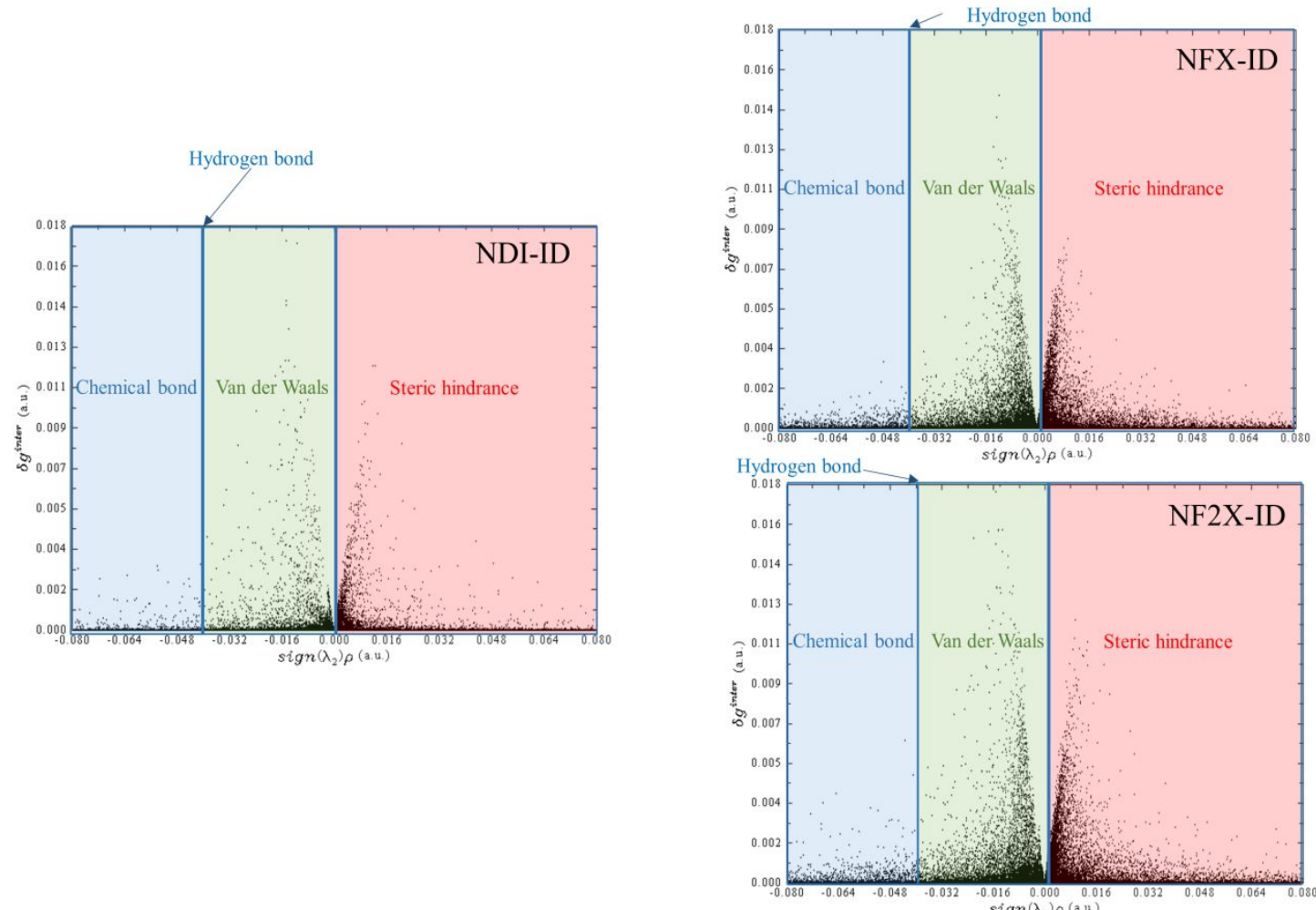

Figure S2 Independent gradient model (IGM) scatter plots of $\mathrm{MAPbI}_{3} / \mathrm{NDI}-\mathrm{ID}, \mathrm{MAPbI}_{3} / \mathrm{NFX}-\mathrm{ID}$ and $\mathrm{MAPbI}_{3} / \mathrm{NF} 2 \mathrm{X}-\mathrm{ID}$ interfaces. 


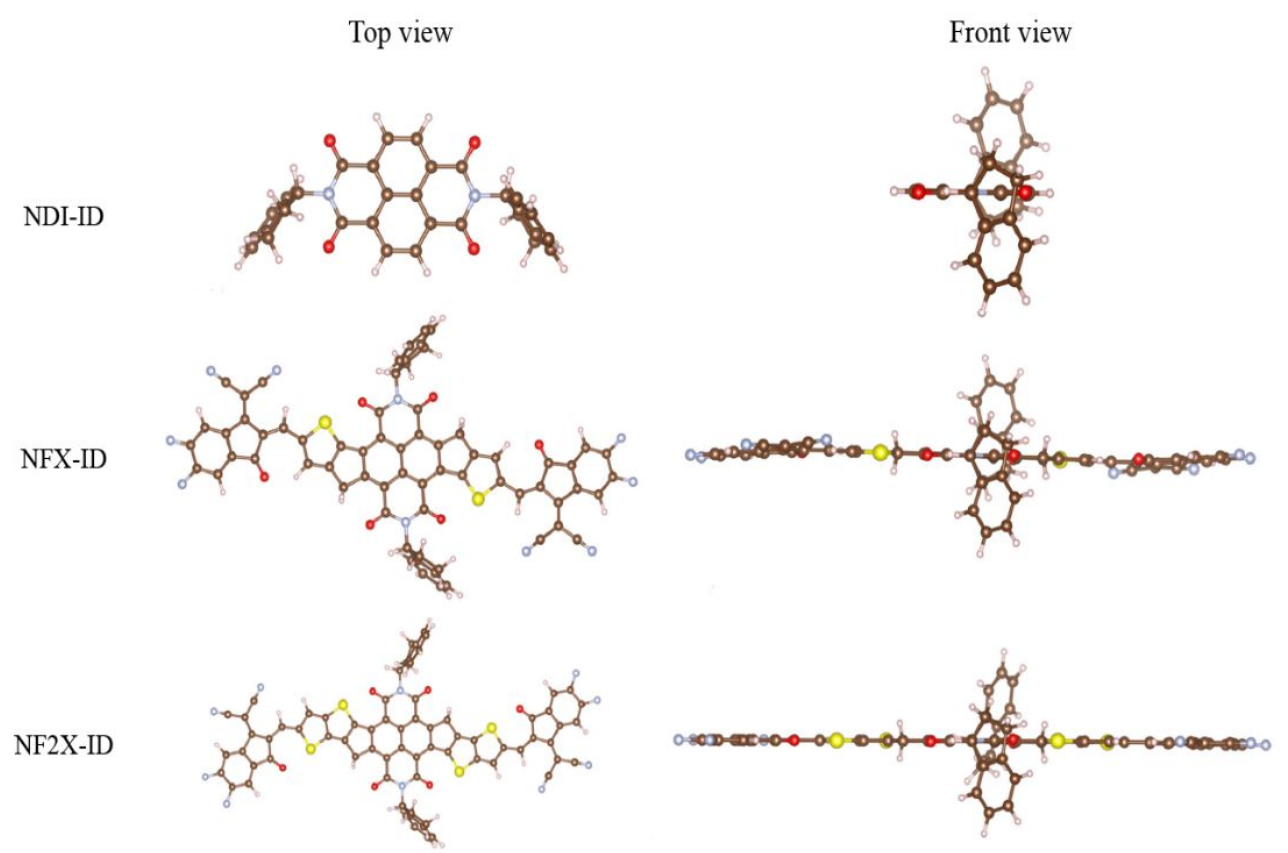

Figure S3 The optimized geometries of the studied molecules at the B3LYP/6-311G (d, p) level.
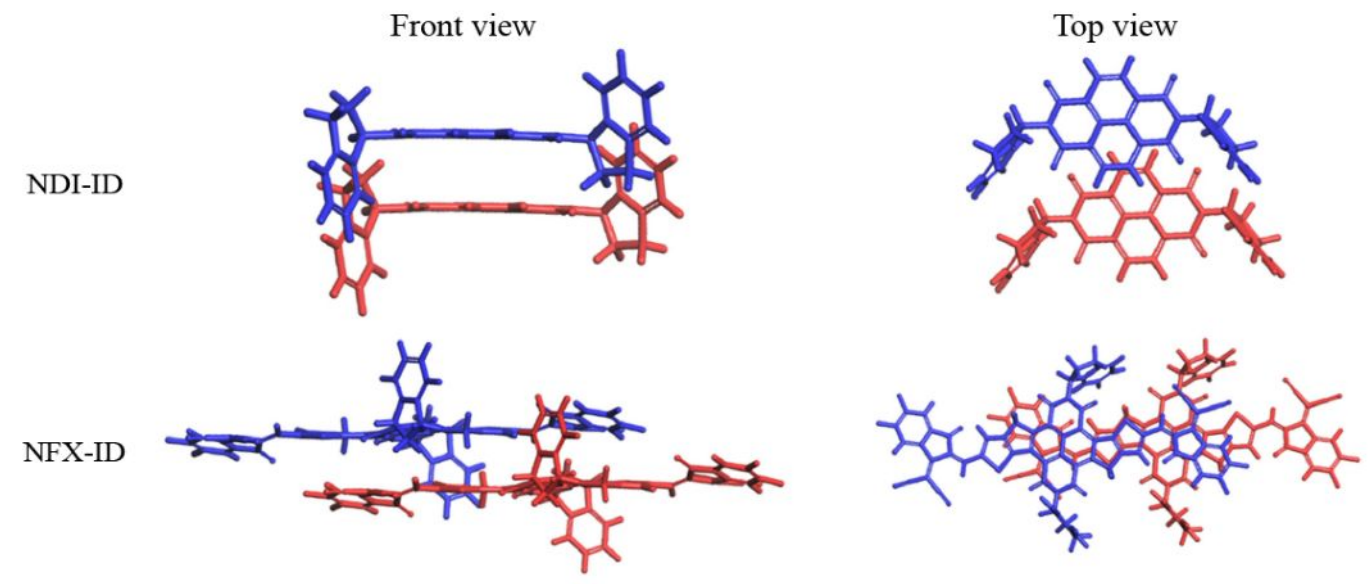

NF2X-ID
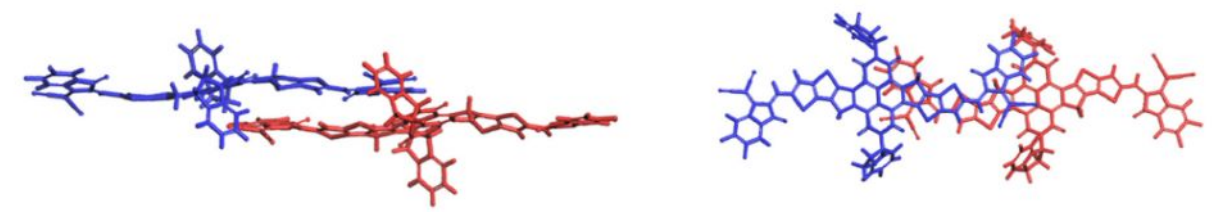

Figure S4 The main stacking modes for all studied molecules. 
NDI-ID
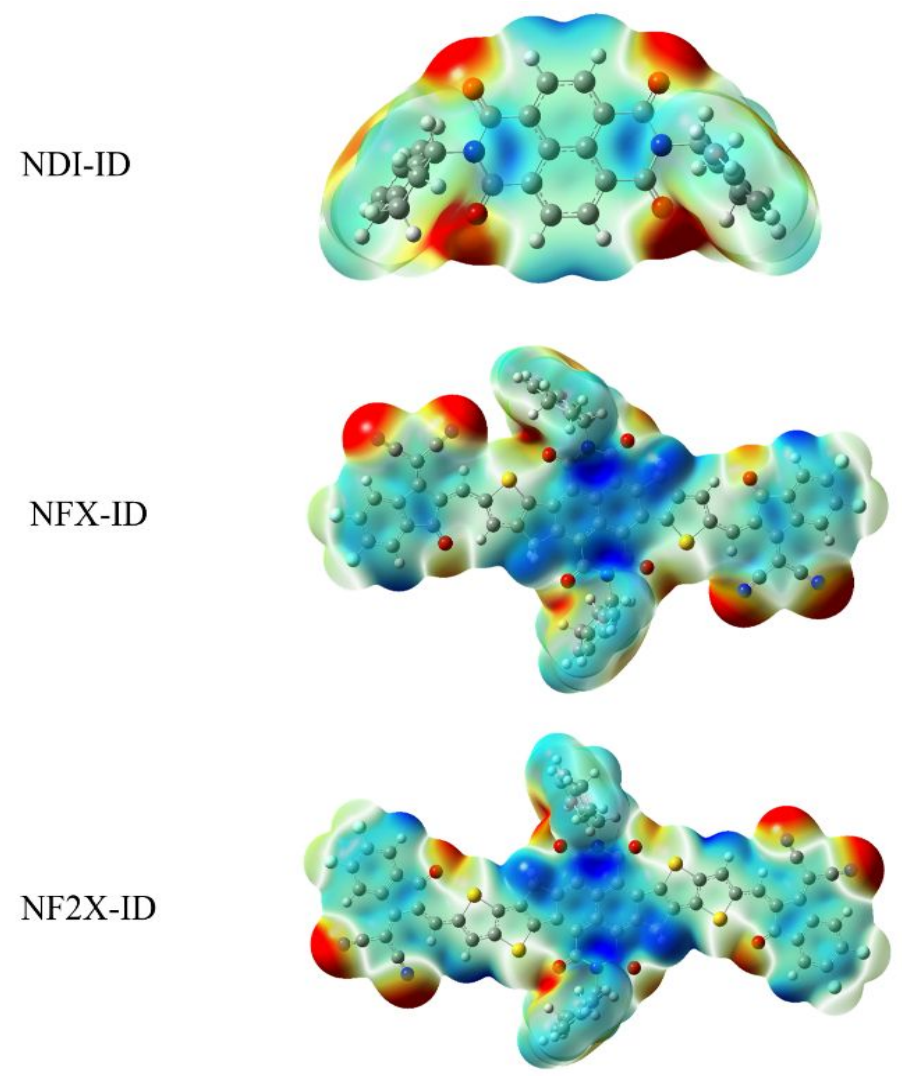

Figure S5 Electrostatic potentials mapped on a surface of total electron densities.

\section{References}

(1) Jung, S. K.; Heo, J. H.; Lee, D. W.; Lee, S. C.; Lee, S. H.; Yoon, W.; Yun, H.; Im, S. H.; Kim, J. H.; Kwon, O. P., Adv. Funct. Mater. 2018, 28, 1800346. 